\title{
Comparison of nifedipine (retard formulation) and mefruside in the treatment of mild to moderate hypertension-a prospective randomized double-blind crossover study in general practice
}

\author{
A. P. DOUGLAS-JONES* \\ M.A.(Camb), M.R.C.S., L.R.C.P. \\ A. D. MITCHELL $\dagger$ \\ B.Sc.(Eng), A.C.G.I., M.B., B.S. \\ *The Surgery, 1 Maid Marion Road, Tuckswood, Norwich and †Late of Medical Department, Bayer (U.K.) Ltd, \\ Newbury
}

\begin{abstract}
Summary
Twenty-two patients under general practice care, suffering mild to moderate hypertension and receiving no active treatment had three baseline blood pressure measurements taken during a single blind 4 week placebo run-in period. One patient was secondarily excluded at this stage because of a placebo response and one patient dropped out for personal reasons. The remaining 20 patients were randomized to receive either nifedipine $20 \mathrm{mg}$ twice a day or mefruside $25 \mathrm{mg}$ once a day in a classical two-period crossover design with 8-week treatment periods separated by a 4-week single-blind placebo washout. During 8 weeks nifedipine therapy the mean supine blood pressure was reduced from 173 $(s . d .=15.4) / 107(s . d .=6.4) \mathrm{mmHg}$ to $150(s . d .=16.7) /$ 93(s.d. $=10.8) \mathrm{mmHg}$ whereas the corresponding reduction for mefruside was from $174(s . d .=15 \cdot 9)$ / $107($ s.d. $=9 \cdot 4) \mathrm{mmHg}$ to $153(\mathrm{s.d}=19 \cdot 1) / 94(\mathrm{s.d} .=9 \cdot 7)$ mmHg. Neither drug affected postural changes in blood pressure. Standing blood pressure measurements under 8 weeks nifedipine therapy fell from $172(s . d .=12 \cdot 3) / 103(s . d .=5.6) \quad m m H g$ to $150(s . d .=17.9) / 94(s . d .=10-0) \quad m m H g$ with corresponding changes for mefruside being 174(s.d. $=14.7)$ / $106(s . d .=9.0) \mathrm{mmHg}$ to $150(s . d .=20 \cdot 2) / 95($ s.d. $=9 \cdot 4)$ mmHg. Since blood pressures returned to within $4 \%$ of baseline values by the end of the placebo washout period it can be inferred that each therapy was a significant $(P<0.05$ for all blood pressure variables) antihypertensive treatment in its own right.
\end{abstract}

KEY WORDS: diabetes mellitus, hypokalaemia.

\section{Introduction}

At the present time, step care therapy for hypertensive patients is widely accepted (Bannan, Beevers and Wright, 1981) with the choice for first line therapy being either a thiazide diuretic or a beta-adrenocep- tor blocking agent. Thiazide diuretics have been linked with gout, potassium loss and maturity onset diabetes and are not usually selected for long-term therapy unless a beta-blocking agent is contraindicated as for older patients, black patients, those with heart failure, obstructive airways disease or brittle diabetes (Bannan et al., 1981).

The calcium antagonist, nifedipine, formulated as a controlled release preparation (Adalat Retard) is effective when administered twice daily (Gould et al., 1982), and does not have the side effect profile of thiazide diuretics (Maeda et al., 1982) nor the restrictive contra-indications of beta-blocking drugs. Initial headache and facial flushing are the main side effects of nifedipine therapy.

Nifedipine should therefore be considered for first line therapy providing comparable efficacy is demonstrated with established treatments, i.e. thiazide diuretics and beta-blocking drugs.

Mefruside is a thiazide-like diuretic with an unusually potent antihypertensive effect, equivalent (when given $25 \mathrm{mg}$ daily) to atenolol $(100-400 \mathrm{mg}$ daily) over a period of 18 months (Sivertsson, Andersson and Hansson, 1979). Furthermore, it is claimed that potassium and metabolic disturbances are minimal (Henningsen et al., 1980) and short- and long-term tolerance good.

As first line antihypertensive therapy is often initiated by the general practitioner, it was appropriate to conduct this study in a general practice setting.

\section{Materials and methods}

The study was randomized using a double-blind, double dummy crossover design with 4-week placebo run-in and washout phases and 8-week treatment periods. During the treatment periods patients received either $25 \mathrm{mg}$ mefruside daily at $8 \mathrm{a} . \mathrm{m}$. or 
nifedipine $20 \mathrm{mg}$ at 8 a.m. and 8 p.m. together with matching placebo to the alternative therapy.

Twenty-two patients with untreated or unsatisfactorily treated hypertension entered having given informed consent. They had three consecutive baseline supine blood pressure measurements in the range systolic >160 mmHg, diastolic (phase V) 95-120 mmHg. During this placebo run-in phase blood pressure measurements were taken at 2-weekly intervals. By the end of the run-in period one patient had withdrawn for personal reasons and a second was excluded as a placebo responder.

Of the remaining 20 patients, 15 were female and 5 male and their ages ranged from 50-62 years (mean 58 years). None suffered from heart failure, angina pectoris, or a serious systemic disorder. One patient was found to have diabetes mellitus which proved satisfactorily controlled by diet.

The patients attended every 4 weeks over the subsequent 20 weeks and on each occasion blood pressure was measured together with heart rate and weight.

At each patient attendance the practice nurse recorded the blood pressure using a Hawksley random zero sphygmomanometer after the patient had been lying for $5 \mathrm{~min}$ and then on standing. The same procedure was repeated (A.P.D-J.) before comparing the results, which were then averaged. As well as enquiring after coincidental illness and concomitant medication the patient was asked specifically 'Did your treatment upset you?'. A description and rating of evoked adverse effects was then entered onto the record sheets. An assessment of compliance was made on each occasion by counting returned tablets. Serial biochemical measurements were made throughout the study.
For each of the blood pressure variables, interna validation of the crossover design was performedo according to the method described by Armitage and Hills (1979). Standard $t$-tests were used for hypo thesis testing.

\section{Results}

The distributions of relevant pre-entry variable are shown in Table 1. There were no significant differences between those receiving either drug first. The validity of the crossover study was established with respect to the blood pressure variables there $\overrightarrow{.}$ being no discernable period or interaction effect. (calculations for the supine diastolic measurements are available from the authors). Therefore botk treatment periods were combined in reporting the results.

The effects of nifedipine and mefruside on systoli and diastolic blood pressure, both standing and lyings are shown in Fig. 1.

Over an 8-week treatment period under nifedipine therapy, the mean supine blood pressure was re duced from 173(s.d. $=15 \cdot 4) / 107($ s.d. $=6.4) \mathrm{mmHg}$ to 150 (s.d. $=16.7$ ) $/ 93$ (s.d. $=10.8) \mathrm{mmHg}$ whereas the corresponding reduction for mefruside was from $174($ s.d. $=15.9) / 107($ s.d. $=9.4) \quad \mathrm{mmHg}$ to $153(\mathrm{~s} d \overrightarrow{\mathrm{d}}$ $=19 \cdot 1) / 94($ s.d. $=9.7) \mathrm{mmHg}$. Standing blogos pressure reductions for the same period were; nife: dipine $172($ s.d. $=12.3) / 103($ s.d. $=5.6) \quad \mathrm{mmHg} \exists$ $150($ s.d. $=17 \cdot 9) / 94($ s.d. $=10.0) \mathrm{mmHg}$ and mefrusides 174 (s.d. $=14.7) / 106$ (s.d. $=9.0) \mathrm{mmHg}$ to $150($ s.do $=20.3) / 95$ (s.d. $=9.4) \mathrm{mmHg}$. $\quad(P<0.05$ for alp blood pressure variables). There were no significan differences between changes in standing and lyin $\overrightarrow{\vec{S}}$ blood pressures nor any significant effect of eithē̄

TABLE 1. Pre-entry variables of 20 hypertensive patients according to order of treatments [mean (s.d.)]

\begin{tabular}{lcc}
\hline & $\begin{array}{c}\text { Nifedipine/mefruside } \\
(n=10)\end{array}$ & $\begin{array}{c}\text { Mefruside/nifedipine } \\
(n=10)\end{array}$ \\
\hline Males & 1 & 4 \\
Females & 9 & 6 \\
Age (years) & $59 \cdot 5(3 \cdot 4)$ & $57 \cdot 0(4 \cdot 2)$ \\
Weight (kg) & $75 \cdot 2(3 \cdot 2)$ & $76 \cdot 05(5 \cdot 4)$ \\
Duration of disease (years) & $4 \cdot 76(6 \cdot 1)$ & $2 \cdot 02(2 \cdot 7)$ \\
Supine BP (mmHg) & $176.2(9 \cdot 4)$ & $181 \cdot 6(21 \cdot 2)$ \\
Systolic & $107 \cdot 5(5 \cdot 9)$ & $104 \cdot 3(4 \cdot 8)$ \\
Diastolic & $176.9(14 \cdot 2)^{*}$ & $182 \cdot 2(22 \cdot 6)$ \\
Standing BP (mmHg) & $105.6(7 \cdot 2)$ & $102 \cdot 8(5.9)$ \\
Systolic & 3 & 4 \\
Diastolic & 2 & 2 \\
Previous medication & 1 & 2 \\
None & 1 & 1 \\
Diuretics & 2 & 1 \\
Beta-blocker & & \\
Combination & & \\
Others & & \\
\hline
\end{tabular}

$* n=9$. 


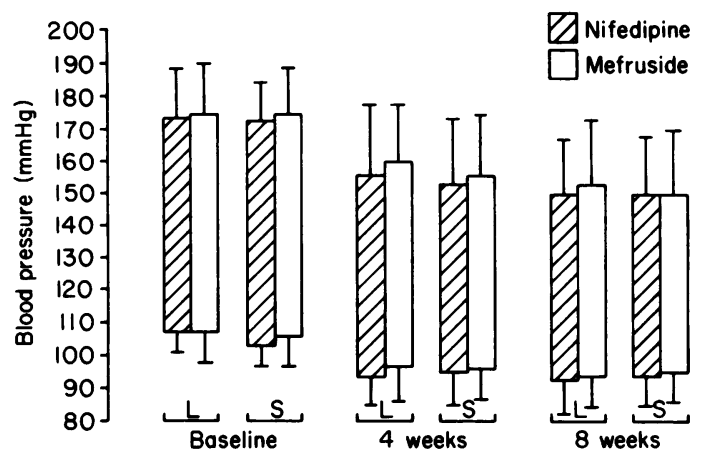

FIG. 1. Effect of nifedipine and mefruside on lying (L) and standing (S) blood pressures after 4 and 8 weeks' treatment. Mean values \pm 1 s.d. Measurements from both treatment periods are combined in Fig. 1 , this being permissible because validation of the crossover design confirmed freedom from period and interaction effects. The baseline values are the immediate pre-treatment values combining measurements obtained at the end of a 4-week single blind placebo run-in period with those at the end of a 4-week single blind placebo washout period for each drug.

drug on heart rate. Weight was unaffected by either drug and did not change with time.

There were no significant differences either within or between treatments with respect to the following biochemical variables: serum sodium, serum potassium, serum calcium, serum uric acid, urea and random blood glucose. However, one patient developed hypokalaemia (serum $\mathrm{K}^{+}=2.9 \mathrm{mmol} / \mathrm{l}$ ) on mefruside. All other patients maintained a serum potassium concentration above $3.5 \mathrm{mmol} / 1$ on both therapies.

The evoked patient complaints and recorded side effects are shown in Table 2.

TABLE 2. Evoked patient complaints and reported side effects

\begin{tabular}{lcc}
\hline Evoked complaints/side effects & Nifedipine & Mefruside \\
\hline Headaches & 3 & 2 \\
Breathlessness & 1 & 1 \\
Nocturnal frequency & 0 & 1 \\
Weight gain & 1 & 0 \\
Dizziness & 1 & 0 \\
Sleepiness & 0 & 1 \\
Swollen ankles & 1 & 0 \\
Numbness in hands & 0 & 1 \\
Facial pain & 0 & 1 \\
Total & 7 & 7 \\
\hline
\end{tabular}

No patient suffered any serious adverse effects and all evoked complaints were recorded as tolerable.

\section{Discussion}

Both nifedipine and mefruside are effective antihypertensive therapies in their own rights as evidenced by the respective blood pressure lowering effects with time. This statement is strengthened by the observation that blood pressures returned to within $4 \%$ of pre-treatment levels by the end of the placebo washout phase. There was no significant difference between therapies in respect of systolic and diastolic blood pressure variables but the question remained as to their comparability. The crossover technique was validated showing no period or interaction effects before combining the therapeutic responses from both treatment periods. Following this a particular variable of interest, the supine diastolic blood pressure, was analysed and it was determined that the treatments could be considered equivalent within 6.9 $\mathrm{mmHg}$ and with a power of $80 \%$.

This claim though modest, was only possible by investing effort initially in reducing observer error, conducting the study in a double-blind fashion, removing subjective error from blood pressure measurements and validating the crossover design afterwards. To establish more equal efficacy between two powerful antihypertensive agents, i.e. to within 5 $\mathrm{mmHg}$ with a power of $95 \%$, would require approximately 60 patients. Thus, nifedipine and mefruside are shown to have a reasonably comparable antihypertensive effect.

The biochemical profiles were not significantly affected by either drug during the relatively short treatment periods. This supports the claim that potassium and metabolic disturbances are minimal with mefruside. The evoked side effects were tolerable, none necessitating withdrawal from the study.

In conclusion, nifedipine, taken twice daily as the controlled release formulation, is an effective antihypertensive agent with an efficacy comparable to the potent thiazide-like diuretic, mefruside. To date, nifedipine has not been implicated in long-term metabolic disturbances sometimes associated with thiazide diuretics.

Nifedipine can also be used in circumstances in which beta-blockers are contra-indicated. This study suggests that nifedipine may therefore be considered as a safe and effective first-line therapy of mild to moderate hypertension in the general practice setting.

\section{Acknowledgment}

We wish to acknowledge the professional services of $\mathrm{Mr} \mathrm{L}$. Dineen, F.I.S., in undertaking the statistical analysis.

\section{References}

Armitage, P. \& Hills, M. (1979) The two-period cross-over clinical trial. British Journal of Clinical Pharmacology, 8, 7 .

BANNAN, L.T., BEEVERS, D.G. \& WRIGHT, N. (1980) ABC of blood pressure reduction: drug treatment. British Medical Journal, 281, 982.

Gould, B.A., Hornung, S.M., Balasubramanian, V. \& RafT- 
ERY, E.B. (1982) Slow channel inhibitor verapamil and nifedipine in the management of hypertension. Journal of Cardiovascular Pharmacology, 4, S369.

Henningsen, N.C., Bergengren, B., Malmborg, O., Pihl, O., RenMarker, K. \& STRAND, L. (1980) Effects of mefruside treatment in hypertension. Acta Medica Scandinavica, 208, 273.

Maeda, K., Tanaka, C., Tsukano, Y., Minamikawa, H., KoMATSU, H., Kotsumi, K. \& INOUE, E. (1982) Antihypertensive effects of the calcium antagonistic agent nifedipine. Drug $R e$
search, 32, 267 .

Sivertsson, R., ANDersson, O. \& Hansson, L. (1979) Blood pressure reduction and vascular adaptation. Acta Medica Scandic. navica, 205, 477.

(Accepted 15 February 1984) 